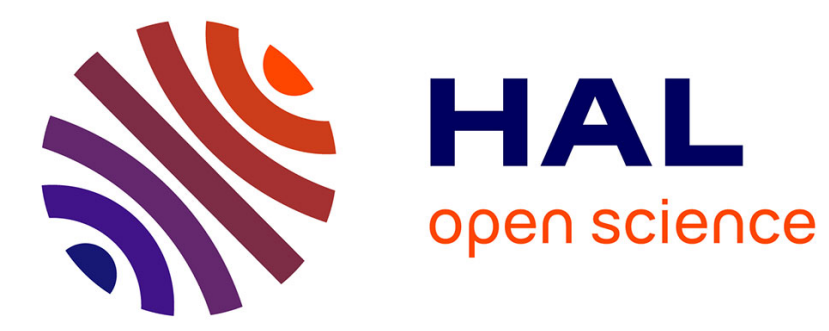

\title{
AK-ILS: An Active learning method based on Kriging for the Inspection of Large Surfaces
}

Antoine Dumas, Benjamin Echard, Nicolas Gayton, Olivier Rochat, Jean-Yves Dantan, Sjoerd van Der Veen

\section{- To cite this version:}

Antoine Dumas, Benjamin Echard, Nicolas Gayton, Olivier Rochat, Jean-Yves Dantan, et al.. AKILS: An Active learning method based on Kriging for the Inspection of Large Surfaces. Precision Engineering, 2013, 37 (1), 10.1016/j.precisioneng.2012.07.007 . hal-02519426

\section{HAL Id: hal-02519426 https://hal.science/hal-02519426}

Submitted on 26 Mar 2020

HAL is a multi-disciplinary open access archive for the deposit and dissemination of scientific research documents, whether they are published or not. The documents may come from teaching and research institutions in France or abroad, or from public or private research centers.
L'archive ouverte pluridisciplinaire HAL, est destinée au dépôt et à la diffusion de documents scientifiques de niveau recherche, publiés ou non, émanant des établissements d'enseignement et de recherche français ou étrangers, des laboratoires publics ou privés. 


\title{
AK-ILS: An Active learning method based on Kriging for the Inspection of Large Surfaces
}

\author{
Antoine Dumas ${ }^{\mathrm{a}, *}$, Benjamin Echard ${ }^{\mathrm{a}}$, Nicolas Gayton ${ }^{\mathrm{a}}$, Olivier Rochat ${ }^{\mathrm{b}}$, \\ Jean-Yves Dantan ${ }^{c}$, Sjoerd Van Der Veen ${ }^{\mathrm{d}}$
}

a Clermont Université, Institut Français de Mécanique Avancée, Institut Pascal, Campus de Clermont-Ferrand les Cézeaux, BP 265, 63000 Clermont-Ferrand, France ${ }^{\mathrm{b}}$ CIMPA S.A.S. (EADS), Centreda, 4 Avenue Didier Daurat, 31700 Blagnac, France

' Arts et Métiers ParisTech, CER de Metz, 4 rue Augustin Fresnel, 57078 Metz Cedex, France

d AIRBUS Operations S.A.S., 316 Route de Bayonne, 31060 Toulouse Cedex, France

\section{Contents}

Tolerance verification permits to check the product conformity and to verify assumptions made by the designer. For conformity assessment, the uncertainty associated with the values of the measurands must be known. In fact, to evaluate form characteristics of large aircraft structure workpieces, sampling is required, so a measurement error is present: exact estimation of form characteristics would require complete knowledge of the surface. To minimise this measurement error, this paper presents a Krigingbased procedure to identify the minimum of measured points to check the conformity with a given confidence level. The proposed method is validated on a simple example of orientation tolerance and then performed to inspect the form defect on three large aircraft workpieces.

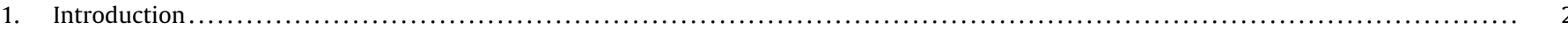

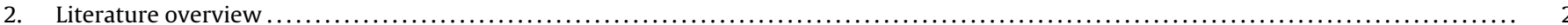

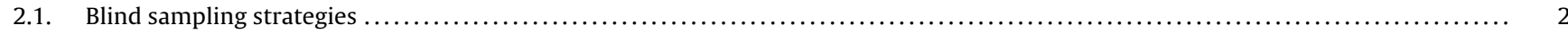

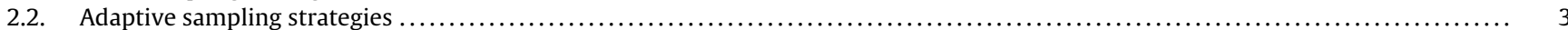

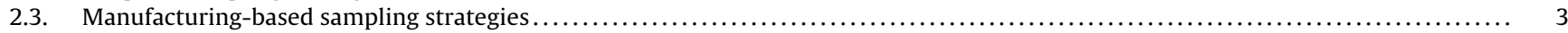

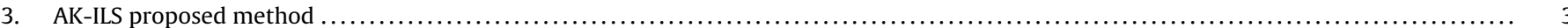

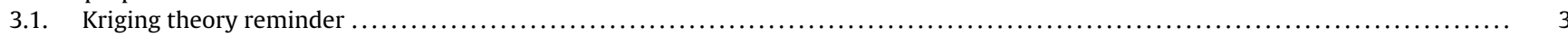

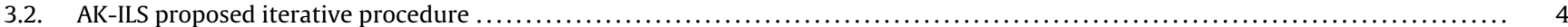

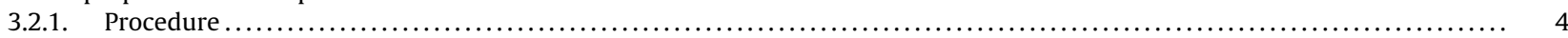

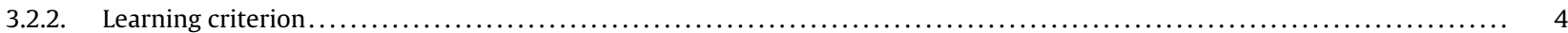

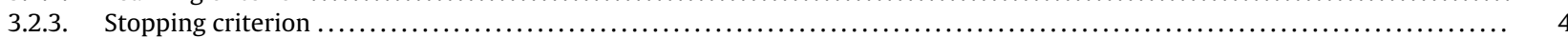

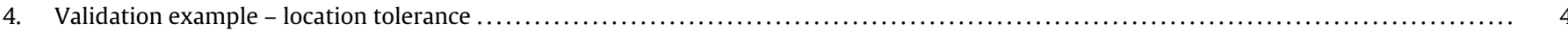

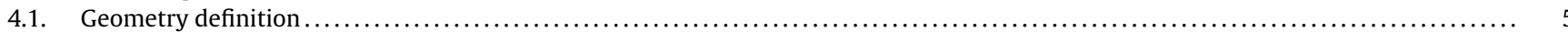

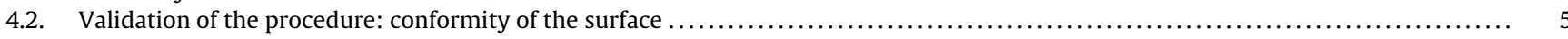

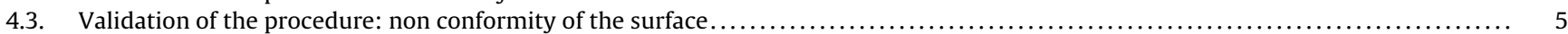

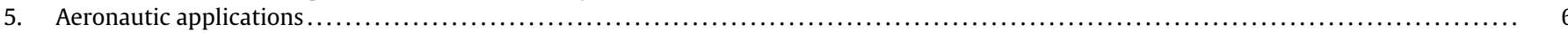

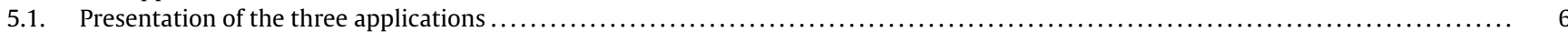

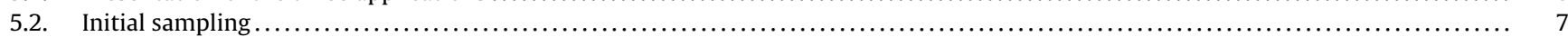

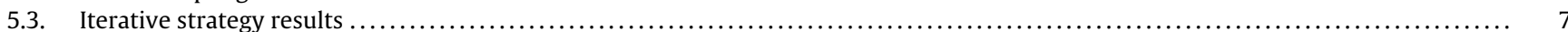

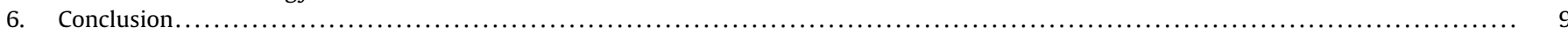

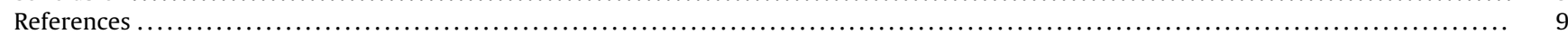




\section{Introduction}

As technology increases and performance requirements continually tighten, the cost and required precision of assemblies increase as well. There is a strong need for increased attention to tolerance design to enable high-precision assemblies to be manufactured at lower costs. Tolerance verification permits to check the product conformity and to verify assumptions made by the designer. Tolerance verification defines inspection planning and metrological procedures for functional requirements, functional specifications and manufacturing specifications.

Metrology always aims at providing reliable information as a basis for decisions. Measurement results are affected by measurement uncertainty and measurement error, which lead to technical and economic risks in industrial companies. By assessing the risks and the connected consequences of the decisions (conformity verification), the significance of the measurement result can be evaluated [1]. In the ISO TS17450-2 [2], the notion of uncertainty is generalised to specification and verification. The uncertainties through the product life cycle span from the design intent to the inspection activity. The uncertainty is divided into correlation uncertainty, specification uncertainty and measurement uncertainty [3]. The uncertainty in measurement constitutes a parameter, associated with the result of a measurement, which characterises the dispersion of the values that could reasonably be attributed to the measurand (GUM - "Guide to the expression of uncertainty in measurement" [4]). The uncertainty of measurement allows establishing an interval around the best estimate that contains the true value of a measurand with a stated level of confidence, e.g. $95 \%$.

Moreover, an exact estimation of measurand without measurement uncertainty would require complete knowledge of the surface. Using a Coordinate Measuring Machine (CMM), the knowledge of the surface is reduced to a set of points which depends on the sampling strategy. In order to verify conformity with a CMM, sampling is required, so a measurement error is always present.

In the context of aircraft industry, the conformity verification is an important issue. We can illustrate this:

- Generalisation of new materials to all aero structures: the carbon fibre composites now represent over $50 \%$ of the structural mass of the A350-XWB and the B787 "Dreamliner". Their implementation, however, is difficult and involves in an actual industrial know-how to avoid the proliferation of fatal defect in the manufactured parts.

- Tendency to replace some assemblies, sometimes consisting of several hundred components, with a large one-piece structure: the innovative design of the B787, depending on the "full barrel" technology where each section of the fuselage is coated with a unique carbon skin produced by filament winding and cured around a mandrel having both shape and size of said section, provides an excellent illustration. Airbus is not outdone and also carries out R\&T programs on this subject for its next generation of fuselage. This change is justified by the need to design ever more efficient structures at the lowest cost. However, their large size complicates the conformity verification.

- Generalisation of the "extended enterprise", which is to delegate to partner companies, having their own design desk and production facilities, the full realisation of whole sections of the aircraft structure. It raises the issue of conformity verification with contractual deliverables and resources to implement to inspect it.

Moreover, a brief discussion of this issue is given by Weckenmann et al. [5]: "In production metrology workpieces are inspected by measuring their specified characteristics. The generated measurement results are used as a basis for decisions for conformity assessment, process evaluation and statistical process control. Regardless of the economic value of measurement results as a decision base and due to the fact that the benefits through quality inspections in industrial enterprises are mostly hidden, production metrology is often considered merely as source of expenses. A verifiable proof of the economic value of measurements and the optimization of the ratio between value and expenses of measurement systems failed until now because of the missing monetary evaluation of the value of measurements".

This paper focuses on the measurement error due to the sampling strategy (one of the possible uncertainty sources in a CMM based measurement) and the expenses of measurement process. That is why it is important, when sampling strategy is defined, to take into account their impacts on the measurement cost and measurement error. These two concepts (measurement cost and measurement error) are usually considered as conflicting goals.

This paper presents an original approach, called AK-ILS for "Active learning method based on Kriging for the Inspection of Large Surfaces". It is based on an optimal estimation technique Kriging - already widely implemented in other areas of science and industry, which has been tending in recent years to become widespread in the world of mechanics. Section 2 of this paper presents a critical overview of the literature and discusses the pros and cons of the main identified sampling strategies (blind, adaptive and manufacturing-based) that are intended for inspection and reconstruction of surfaces. After some theoretical notions on Ordinary Kriging, the principles of this new method AK-ILS are exposed in Section 3. The validation of the method on a digital test case and its application to actual industrial cases (three aircraft structural parts) are respectively described in Sections 4 and 5. A conclusion ends this paper.

\section{Literature overview}

Using a CMM implies to define a number of measures. However, the relevant sampling strategy is not supplied within the feature control frame. So, it seems to be function of the operator and the machine capacity. Due to economic reason or because measurements are time-consuming, main geometric elements (planes, spheres, cylinders, straight lines, circles, etc.) may be measured with few points. However, it is also possible to have a lot of measures. In this case, the questions about their locations and the required number rise.

The literature overview can be divided into three main groups based on the categories introduced by Colosimo et al. [6]. First, the inspections can be performed directly on a design of experiments obtained using a blind sampling strategy. Secondly, methods rely on adaptive strategies where the design of experiments is iteratively improved. The third group relates to provide a manufacturingbased design of experiments. A discussion about the choices made for the proposed procedure is formulated in Section 3.

\subsection{Blind sampling strategies}

Three methods are presented here: uniform sampling, Latin Hypercube Sampling and Hammersley sequence. A uniform random selection can be performed to select the measurements points. $m$ points are chosen, following a uniform law in the area of study. However, this method is not efficient, in particular in the case of small number of measures. A Latin Hypercube Sampling is a spacefilling design, it was first proposed by McKay et al. [7]. This is the generalisation of the Latin Square in all dimensions. The main characteristic is that the projection of the samples on each axis of the hyperplane is uniform. However, it may happen a LHS is not really a space-filling design if it is not coupled to a distance-based criterion. Lee et al. [8] have developed a sampling method which significantly 
reduces the required number of measures for a given accuracy. Their sampling strategy is based on the Hammersley sequence. The latter is very relevant because it approaches the lower bound of the discrepancy defined by Roth [9]. The statistic concept of discrepancy can be seen as a residual error. The lower bound is thus the minimum residual error which can be reached [8].

The measured data are usually processed using methods like the minimum zone $[10,11]$ or the least squares [11] but they often underestimate the actual deviation of the toleranced surface. Prieto et al. [12] present a method to build a tolerance zone using NURBS (Non-Uniform Rational Basis Splines) and the distance between measures and CAD (Computer-Aided Design) model. The first step is to match both measurements and CAD model in the same Cartesian coordinate system. It amounts to find the $3 \mathrm{D}$ rigid transformation to be applied on one of the shape. Then, a NURBS surface is built based on CMM data. The perpendicular distance between the measured points and the CAD model is used to compute a tolerance interval. Indeed, the distribution of the distance provides a standard deviation $\sigma$. The tolerance zone is computed at $\pm 2 \sigma$ from the NURBS surface. The deviation is then equal to $4 \sigma$. The distance calculation is a particularity which will be reused in the proposed procedure.

\subsection{Adaptive sampling strategies}

An adaptive sampling strategy usually needs a metamodel in order to predict the whole surface shape based on few measured points. Then, a criterion selects the next point to be measured and added to the design of experiments. The metamodel enables to get more information than methods only based on measurements data. Edgeworth and Wilhelm [13] propose an iterative method based on the error profile which is the orthogonal distance between the actual coordinate of the point and the nominal geometry. They fit the error profile with splines and then select the next point to be measured where the interpolation curve is the most deviated from the nominal curve. Working with the error profile enables the methodology to be free from the nominal geometry which is more consistent. Badar et al. [14] propose an optimization search method for reducing the sample size, it is called the Tabu search. The next selected point is given by the algorithm.

Pedone et al. [15] developed a Kriging-based method to define iterative design of experiments. In their paper, Kriging is used to model the shape of the toleranced element. They use the specific features of Kriging to define three different learning criteria and then compare the results of each criterion between themselves. The iterative improvement of the design of experiments stops when enough information is collected to certify the conformity (or not) of the product, or if the maximum number of iterations is reached. Moreover, an initial plan is required; thus they suggest a spacefilling design like Latin Hypercube Sampling (LHS) design, distancebased design or even a uniform design.

\subsection{Manufacturing-based sampling strategies}

Colosimo et al. [6] proposed a manufacturing-based sampling using the specific signature left by the manufacturing process on the machined parts. The best sampling strategy is chosen by minimising the size of the regression-based tolerance interval. The method mainly concentrates points in area where, according to the model of the signature, the deviation from the nominal geometry is the most important. Another method, developed by Mestre and AbouKandil [16], is defined to overestimate the actual form error. They propose to predict the whole surface or profile from a set of CMM data and roughness measurements. A confidence interval is computed using variance values related to predictions. Thanks to this confidence interval, they compute a form error by the minimum zone method. This form error should overestimate the actual one of the toleranced element. Mestre proposes, in his thesis, an iterative enhancement of the design of experiments using the variance as selection criterion, that is why this method can also be part of adaptive sampling.

\section{AK-ILS proposed method}

Methods introduced in the last section are not really suitable to answer the problematic, which is the ability to check the conformity of large free-form surfaces at a least cost. The proposed procedure is not interested in knowing the deviation of the toleranced element but it focuses only on the conformity of the profile. Blind strategies and manufacturing-based strategies are not appropriate for large parts, a too large number of sample points should be required and using specific characteristics of the surface linked to the manufacturing process is too restrictive for large structures. Adaptive strategies are then the best methods to use. Pedone's method [15], based on Kriging, focuses on estimating as far as possible the deviation of the toleranced surface or profile. The form error is computed by the minimum zone method [10,11]. In the case of profile of a surface, this is hardly possible. Secondly, learning criteria used by Pedone et al. are not adapted for the current problematic that is why a specific criterion has to be developed in order to add points where the surface is the most likely outside the tolerance. Moreover, in order to be more consistent and adjustable to different geometries, it is important to work with the orthogonal distance. Kriging presents interesting probabilistic characteristics which are useful to compose a criterion. The proposed procedure AK-ILS takes advantage of several points of the literature review:

- the Hammersley sequence for the initial design of experiments,

- the Kriging metamodel method for its probabilistic properties to compute the selection criterion,

- the metamodel based on the orthogonal distance in relation to the nominal geometry for more consistency and adaptability,

- the iteratively improved design of experiments in order to have a limited number of measurements.

This section is devoted to the proposed iterative procedure based on Kriging. The first subsection is a reminder on the Kriging theory. Then, the iterative procedure is explained in Section 3.2.

\subsection{Kriging theory reminder}

Kriging was first developed for mining applications in the fifties by Krige [17] and then Matheron [18]. Later, this method has gained consideration in the computer experiments field thanks to its stochastic properties and accurate predictions.

In this paper, ordinary Kriging is used. A Kriging model needs a design of experiments to define its stochastic parameters. Then, predictions of the response can be made on an unknown point belonging or not to the design of experiments. Given a design of experiments $\mathbf{X}=\left[\mathbf{x}_{1}, \ldots, \mathbf{x}_{m}\right]$, with $\mathbf{x}_{i} \in \mathbb{R}^{n}$ the $i$ th experiment, and $\mathbf{y}=\left[y_{1}, \ldots, y_{m}\right]$, with $y_{i} \in \mathbb{R}$ the corresponding response to $\mathbf{X}$, at any point $\mathbf{x}$, the prediction value $\mu_{\hat{y}}(\mathbf{x})$ of the actual one $y(\mathbf{x})$ is computed by:

$\mu_{\hat{y}}(\mathbf{x})=\beta+\mathbf{r}(\mathbf{x}) \mathbf{R}_{\theta}^{-1}(\mathbf{y}-\beta \mathbf{1})$

where $\mathbf{r}(\mathbf{x})$ is a vector of the correlation values between the predicted point $\mathbf{X}$ and all points of the design of experiments $\mathbf{X} . \mathbf{R}_{\theta}$ is the correlation matrix of the design points.

Moreover, Kriging provides a confidence index about the prediction. It is called the Kriging variance, this is the mean squared 
error between the prediction $\hat{y}(\mathbf{x})$ and the actual value $y(\mathbf{x})$. It is given by the expression:

$\sigma_{\hat{y}}^{2}(\mathbf{x})=\sigma_{z}^{2}\left(1+u(\mathbf{x})^{t}\left(\mathbf{1}^{t} \mathbf{R}_{\theta}^{-1} \mathbf{1}\right)^{-1} u(\mathbf{x})-\mathbf{r}(\mathbf{x})^{t} \mathbf{R}_{\theta}^{-1} \mathbf{r}(\mathbf{x})\right)$

where $u(\mathbf{x})=\mathbf{1}^{t} \mathbf{R}_{\theta}^{-1} \mathbf{r}(\mathbf{x})-1$.

Kriging is an exact interpolation method, so predictions made at any point $\mathbf{x}$ belonging to the design of experiments give $\hat{y}(\mathbf{x})=$ $y(\mathbf{x})$. Thus, the Kriging variance is null at these points. It becomes important in unexplored areas; it represents the uncertainty of the predictions. This is a good index to improve a design of experiments.

\subsection{AK-ILS proposed iterative procedure}

The goal of the algorithm developed in this section is to be able to inspect at a least cost the conformity of a large geometrical form. The result must be either "the part is in the tolerance with such a level of confidence", or "the part is out of the tolerance at such a coordinate" as fast as possible, i.e., with the least number of measurements. This algorithm does not need to compute the form error.

Before starting the procedure, both CMM data and the reference model need to be adjusted in the same Cartesian coordinate system. The orthogonal distances between the measures and the reference surface (nominal geometry) are computed. The metamodel is based on these distances, i.e., it builds an approximation of the orthogonal distances between the measured points and the nominal points. All distances are depending on the location of the measured points using their coordinates (either only $(x, y)$ or $(x, y, z)$ following the kind of surface). The type of inspected tolerance is the location of a profile of a surface. So in this case, the position of the tolerance zone is known, in particular, it can be located thanks to the nominal geometry. Since the procedure uses the orthogonal distances between the measurements and the nominal geometry, distances can be directly compared to half the tolerance value: a higher value of the distance signals the non conformity of the measurement and a lower value means the conformity of the measurement. A first Kriging model is built from an initial design of experiments; points are iteratively added to the design of experiments. These points are properly chosen following a specific criterion and the Kriging model is then updated in each iteration.

\subsubsection{Procedure}

1. Initialisation

(a) Initial sampling - $m$ initial coordinates $\left(x_{i}, y_{i}\right)_{i=1, \ldots, m}$ are defined following the Hammersley sequence. The third coordinate is measured with a CMM.

(b) Distance - the orthogonal distance between the measured points and the nominal geometry is computed: $\left(d_{i}\right)_{i=1} \ldots m$

(c) Potential points - a grid of $p$ coordinates is defined. These are the candidate points $\left(x_{j}, y_{j}\right)_{j=1, \ldots p}$ which could be measured and added to the design of experiments. The distances for all potential points will be predicted using the Kriging model.

(d) CL (Confidence Level) - this value is given by the operator. The conformity of the surface is asserted with such a Confidence Level; it varies from 0 to 1, 1 being the maximum confidence level.

2. Loop

(a) Kriging model - a Kriging model is built using the $\left(x_{i}, y_{i}\right)_{i=1, \ldots, m}$ coordinates as input data and $\left(d_{i}\right)_{i=1, \ldots, m}$ as response values. In cases where the studied geometry contains vertical parts, the input data are the three coordinates $\left(x_{i}, y_{i}\right.$, $\left.z_{i}\right)_{i=1, \ldots, m}$ (case of the third aeronautic application).

(b) Kriging predictions: predictions give normal random variables:

$\hat{d}\left(x_{j}, y_{j}\right) \sim \mathcal{N}\left(\mu_{\hat{d}}\left(x_{j}, y_{j}\right), \sigma_{\hat{d}}\left(x_{j}, y_{j}\right)\right)_{j=1, \ldots, p}$.

(c) The stopping criterion PBC (ProbaBility of Conformity) is computed (explained after).

(d) Inspection implies two solutions:

- $\mathrm{PBC} \geq \mathrm{CL}$ - the conformity of the surface is asserted with the required confidence interval. No (more) iterations are required: end of the loop.

- $\mathrm{PBC}<\mathrm{CL}$ - a new point must be added to improve it. The learning criterion selects a specific point among all $\left(x_{j}, y_{j}\right)_{j=1 \ldots \ldots}$. This point is measured and added to the design of experiments.

i. The new measured point is within the tolerance zone - go back to 2(a).

ii. The new measured point is outside the tolerance zone - the non conformity of the surface is asserted: end of the loop.

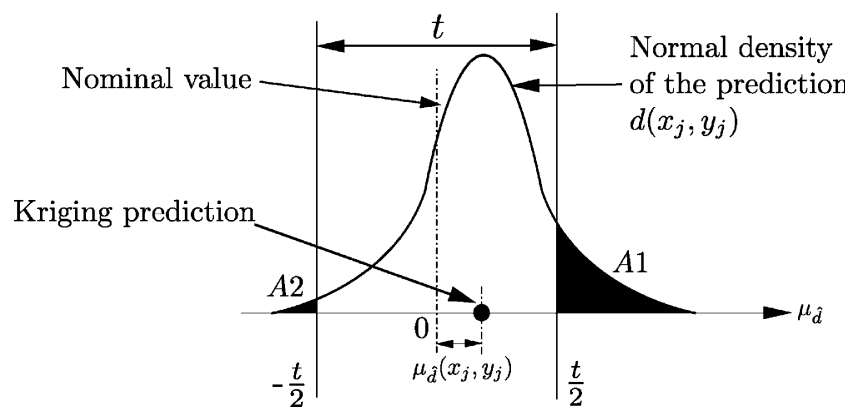

Fig. 1. Diagram showing the probability to be outside the tolerance zone of a Kriging prediction (distance), this is the sum of both black areas at the left and the right side of the tolerance zone.

\subsubsection{Learning criterion}

The learning function determines the best next point to be measured. It represents the active learning aspect of AK-ILS. In tolerance inspection, points having a high potential risk to be outside the tolerance zone must be measured. To answer this problematic, the event $A$ which is "the point is outside the tolerance zone" is defined as:

$A=\left\{\left(d(x, y) \geq \frac{t}{2}\right) \cup\left(d(x, y) \leq-\frac{t}{2}\right)\right\}$

where $t$ is the size of the tolerance of the surface. The criterion selects the point which is the most likely outside of the tolerance zone among the candidates, i.e., which maximises the probability the event $A$ occurs:

$\left(x_{j}, y_{j}\right)=\arg \max _{j=1}^{p}\left\{\operatorname{Prob}\left[A\left(x_{j}, y_{j}\right)\right]\right\}$

with, under Gaussian assumptions:

$$
\begin{aligned}
\operatorname{Prob}\left[A\left(x_{j}, y_{j}\right)\right]= & 1-\Phi\left[\frac{(t / 2)-\mu_{\hat{d}}\left(x_{j}, y_{j}\right)}{\sigma_{\hat{d}}\left(x_{j}, y_{j}\right)}\right] \\
& +\Phi\left[\frac{-(t / 2)-\mu_{\hat{d}}\left(x_{j}, y_{j}\right)}{\sigma_{\hat{d}}\left(x_{j}, y_{j}\right)}\right]
\end{aligned}
$$

where $\Phi$ is the standard cumulative distribution function. It is calculated for all candidate points of the grid defined in step 1.(c) of the procedure. Fig. 1 shows the probability to be outside the tolerance zone for a measure $\left(x_{j}, y_{j}\right)$ belonging to the potential points. It is the sum of the black areas $\left(A_{1}\right.$ and $\left.A_{2}\right)$.

\subsubsection{Stopping criterion}

The stopping criterion $\mathrm{PBC}$ is the probability for all candidate points to be simultaneously within the tolerance. It is therefore the product of the probabilities to be within the tolerance of all candidate points:

$\mathrm{PBC}={ }_{j=1}^{p}\left\{1-\operatorname{Prob}\left[A\left(x_{j}, y_{j}\right)\right]\right\}$

The algorithm stopped when PBC reaches $\mathrm{CL}$, step 2.(d) of the procedure:

$\mathrm{PBC} \geq \mathrm{CL}$.

\section{Validation example - location tolerance}

In this section, the method is validated on a numerical example. A numerical flat surface with defects is created; the example has been built so that the proposed procedure should conclude to the 
conformity of the surface in the first case and to the non conformity in the second case adapting the tolerance value $t$.

\subsection{Geometry definition}

The inspected tolerance is the location of a plane. Considering $\left(x_{j}, y_{j}\right)_{j=1, \ldots, p}$ the coordinates distributed on a square grid where $0 \leq x_{j}, y_{j} \leq 20$, the number of candidate points is set to $p=60^{2}$. Assuming the nominal plane is located at $z=0$, a distance is computed for all coordinates which correspond to the difference between an actual manufactured plane and the nominal plane. The distance $\left(d_{j}\right)_{j=1, \ldots, p}$ is given by the following equation:

$d_{j}=0.05 \cos \left(0.6 x_{j}+4\right)+0.2 U\left(\omega_{j}\right)$

The distance is generated with a macrogeometric default using the cosine function and with some correlated imperfections. $U(\omega)$ is a Gaussian process with an isotropic exponential correlation function of parameter $\theta=20$. For two points $\mathbf{x}_{i}$ and $\mathbf{x}_{j}$, the correlation between them is computed as follows:

$r\left(\mathbf{x}_{i}, \mathbf{x}_{j}\right)=\exp -\frac{\left\|\mathbf{x}_{i}-\mathbf{x}_{j}\right\|}{\theta}$

The tolerance zone is composed of two parallel planes shifted vertically in relation to the nominal plane of the half of the interval tolerance $(t): z_{\text {sup } / \text { inf }}= \pm(t / 2)$. The value of the tolerance interval $(t)$ depends on the studied case.

The coordinates $\left(x_{i}, y_{i}\right)_{i=1, \ldots, 20}$ of 20 initial points are computed using the Hammersley sequence. The 20 closest points of the Hammersley's are picked up among those of the candidate grid.

\subsection{Validation of the procedure: conformity of the surface}

Below are summed up the characteristics of this test case:

- Maximum distance from the nominal surface: $0.13 \mathrm{~mm}$

- $t: 0.29 \mathrm{~mm}$

- CL: 0.997

Fig. 2 shows the contour plot of the distance. The lighter the colour, the farther from the nominal geometry the distance. The white dots are the initial design of experiments.

AK-ILS must add points mainly in the light areas. Fig. 3 shows the simulated Kriging distance based on the final design of experiments. The closest points to the tolerance bound (in the white areas) are added to the design of experiments. On the whole, predictions

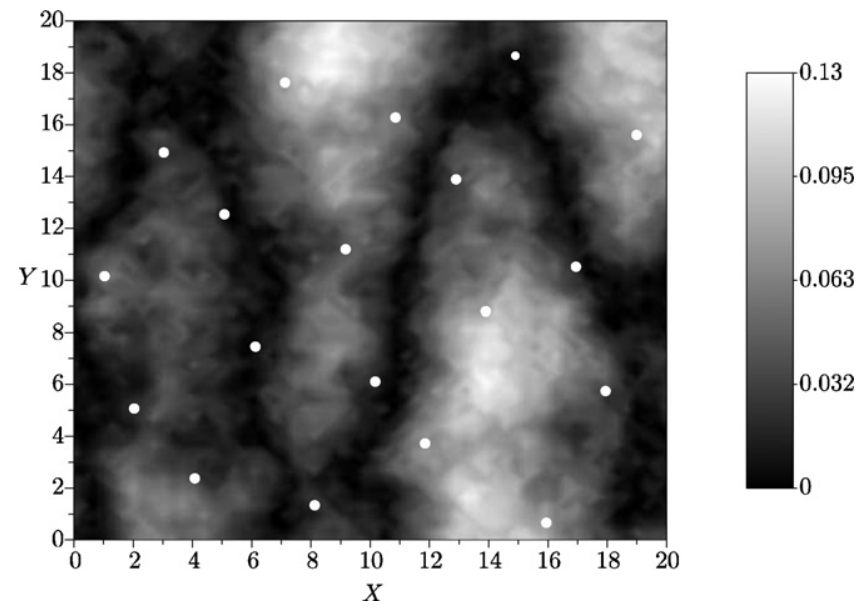

Fig. 2. Numerical distance between the surface and the nominal plane. The initial design of experiments is represented by the white dots. The grey levels correspond to the distance values.

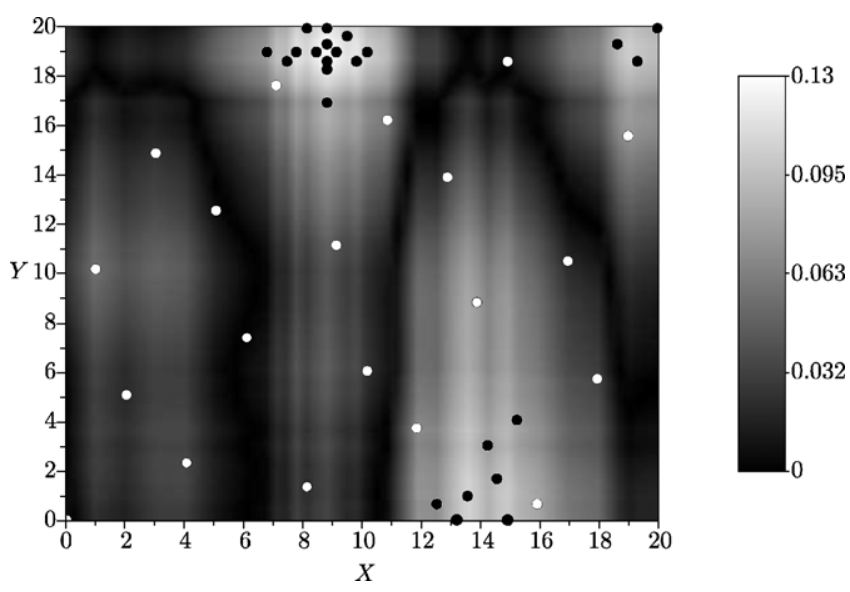

Fig. 3. Final Kriged distance after 25 iterations, the procedure concludes to the conformity by reaching an accuracy of 0.9979 . The grey levels correspond to the distance values.

of the distance are well modelled because no areas remain unexplored. The procedure succeeds in concluding on the conformity of the surface with a probability of $99.79 \%$ after adding 25 points among the 1580 possible sites. The total number of measure is then 45. The convergence of $\mathrm{PBC}$ is shown in Fig. 4.

\subsection{Validation of the procedure: non conformity of the surface}

The same surface as previously is tested, the only difference is about the tolerance value which is decreased in order to get areas outside the tolerance zone. Below are summed up the characteristics of this test case:

- Maximum distance from the nominal surface: $0.13 \mathrm{~mm}$

- $t: 0.25 \mathrm{~mm}$

- Number of points outside the tolerance zone: 15

Fig. 5 shows the contour plot of the distance, the bold contour defined the areas which are outside the tolerance zone. AK-ILS must find a point within one of these areas.

Fig. 6 shows the final Kriged distance after 5 iterations. The added points are focused on the areas at risk. The procedure eventually finds a point (grey diamond) outside the tolerance zone and stops iterating. The procedure succeeds to find one of the 15 points

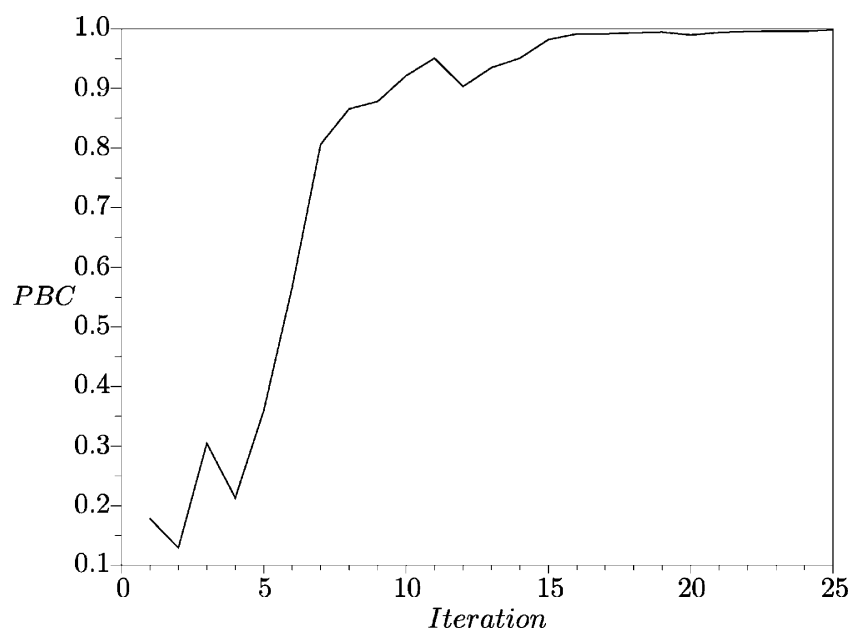

Fig. 4. Convergence of $\mathrm{PBC}$, from 0 (low confidence level) to 0.997 (high confidence level). 


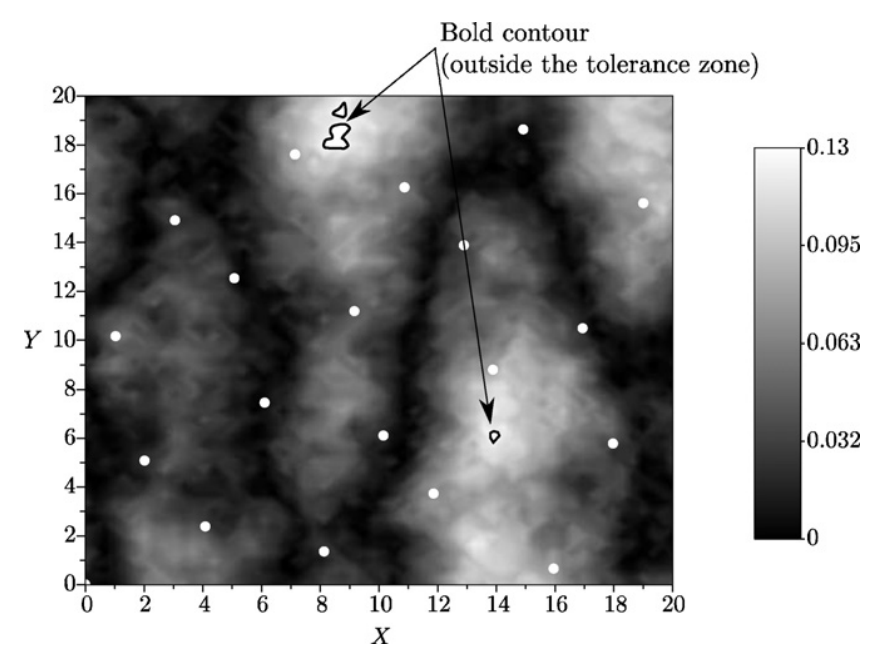

Fig. 5. Distance between the surface and the nominal plane, the initial design of experiments is represented by the white dots and areas outside the tolerance zone by the bold contours. The grey levels correspond to the distance values.

outside of the tolerance after only 5 iterations, the total number of measures is then equal to 25 .

\section{Aeronautic applications}

\subsection{Presentation of the three applications}

The studied structures are three scaled-down research prototypes manufactured in one piece. The first one is a forward pressure bulkhead (Fig. 7). This part is located between the crew compartment and the nose of the plane. It maintains the pressure of the cabin by sealing the forward of the aircraft. Therefore, the product needs to strictly respect the geometrical specifications in order to perform its functions. Inspecting the conformity of such a part is then really important. Fig. 8 shows the real manufactured prototype of the forward pressure bulkhead. This is a raw workpiece that is why the manufacturing accuracy is rather low. The second aircraft structure is the upper part of a cockpit. Fig. 9 shows the manufactured prototype, this is an interesting structure to study because of the free space designed for the pilot visibility. The third aircraft tested part is a landing gear compartment. This structure receives the landing gear of the plane after the take-off. The prototype is smaller than the actual structure, however its dimensions are still substantial: it is contained in a cube of side $500 \mathrm{~mm}$. This

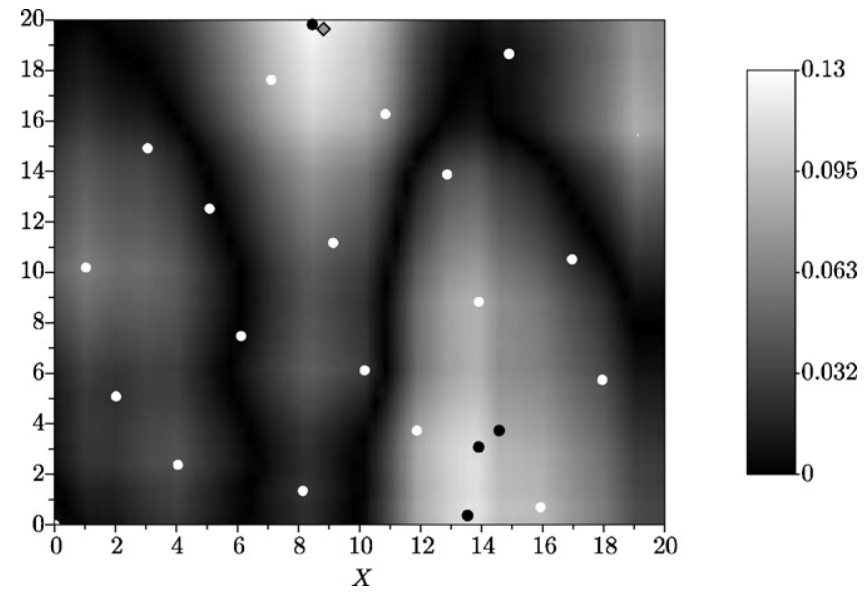

Fig. 6. Final Kriged surface after 5 iterations, the grey diamond is the point outside the tolerance zone. The grey levels correspond to the distance values.

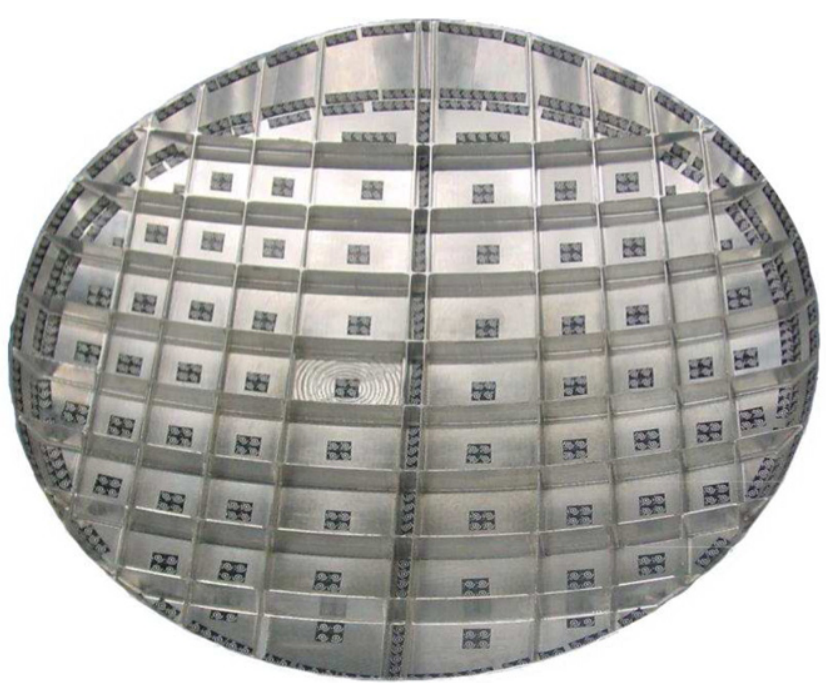

Fig. 7. Prototype of a forward pressure bulkhead.

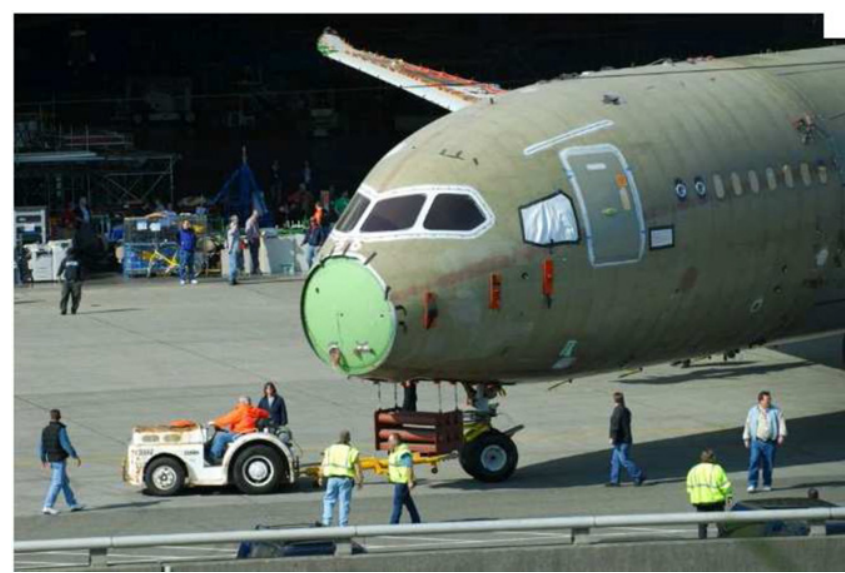

Fig. 8. Forward pressure bulkhead on a civil aircraft being assembled.

part is more complicated than the previous ones. Indeed, this time, vertical parts have to be considered. So some coordinates $(x, y)$ may lead to different surface values $z(x, y)$, see Fig. 10. In order to differentiate them, the three coordinates have to be taken into account in the Kriging model.

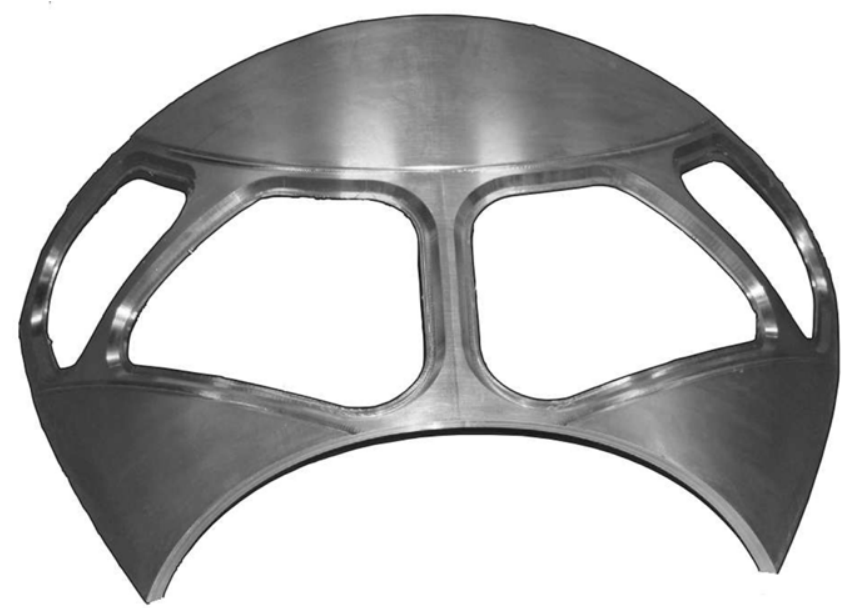

Fig. 9. Manufactured prototype of the cockpit. 


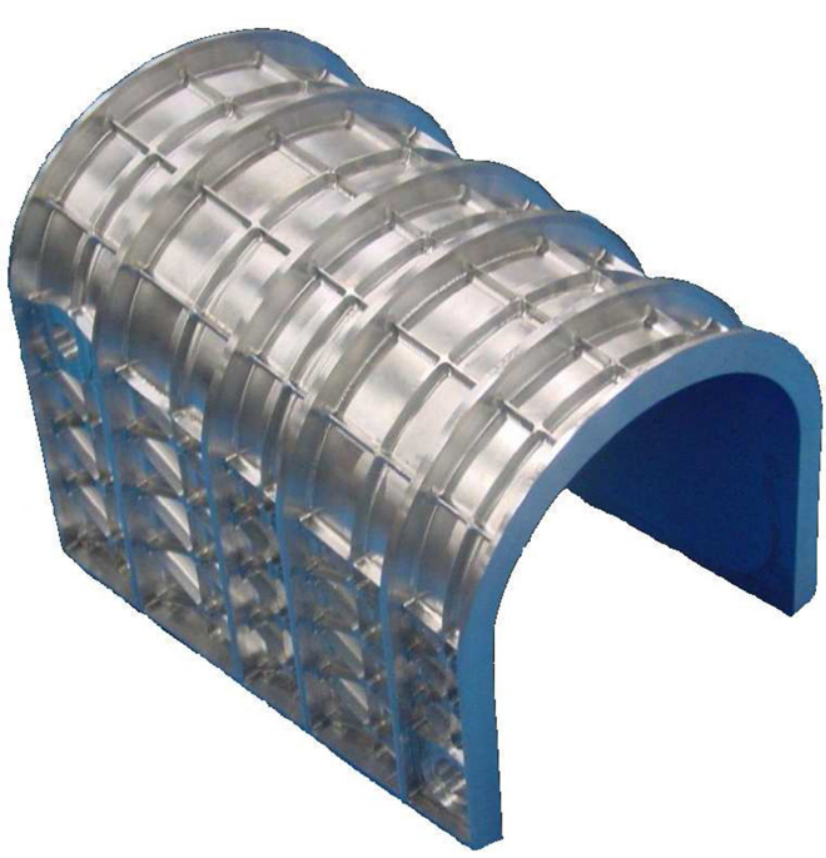

Fig. 10. Manufactured prototype of the landing gear compartment. The studied parts are the inner surfaces of the structure.

Due to the large size of these components, classic inspections are not suitable because they are time-consuming and expensive. The inspected geometrical tolerance is the location of the profile of the surface for all of them.

The three structures are used as industrial test case to validate the method AK-ILS. Indeed, for all of them, defects are created from the nominal geometry in order to have lots of candidate points. The number of candidate points for each part are summed up in Table 1.

\subsection{Initial sampling}

For the three studied structures, a number of 20 initial points are selected to compute the first Kriging model. In order to get a good distribution of these points, they are located using the Hammersley sequence. Then, for each Hammersley points, the nearest points for each structure are selected.

The nominal geometry is assumed to be known, the distances between the measured points and the nominal geometry are computed. After, the initial Kriging model is computed based on these 20 distances and the procedure is launched. Figs. 11-13 show the three Kriged surfaces based on the 20 initial points. The distances are predicted on all candidates points. The colour bar represents the absolute value of the orthogonal distance between the candidate points and the nominal geometry. Figures show that the biggest deviations of the surface in relation to the nominal geometry are in the white areas. The procedure must add points in these areas.

The Kriging model is computed (step 2.(a) of the procedure, Section 3.2) with the coordinates $(x, y)$ for the bulkhead and the cockpit. However, for the landing gear compartment, it is applied using the three coordinates as input parameters in order to differentiate all the points between them.

Table 1

Number $p$ of candidate points for the inspection of each structures.

\begin{tabular}{lll}
\hline Bulkhead & Cockpit & Landing gear compartment \\
\hline 25,000 & 12,000 & 95,000 \\
\hline
\end{tabular}

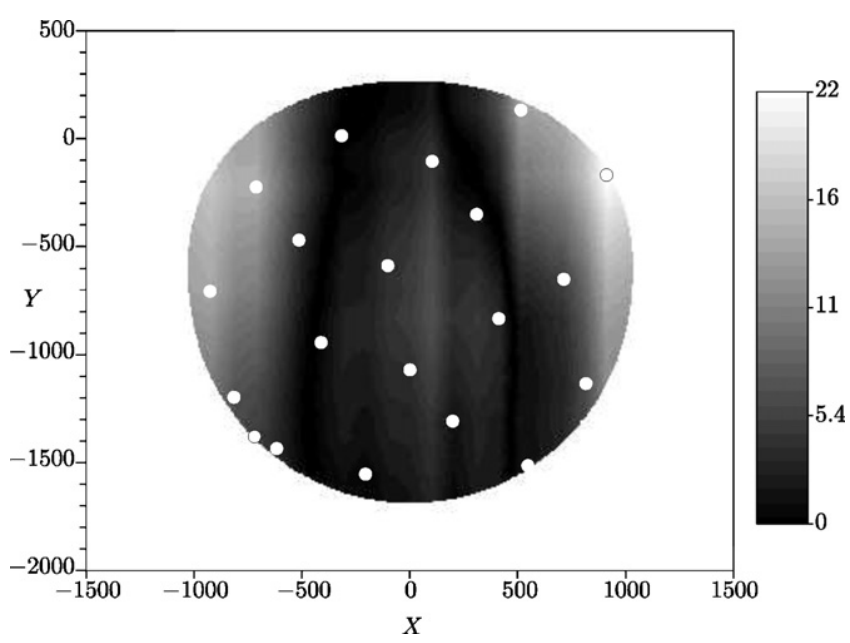

Fig. 11. Initial Kriged bulkhead. White dots are the initial design of experiments The grey levels correspond to the distance values.

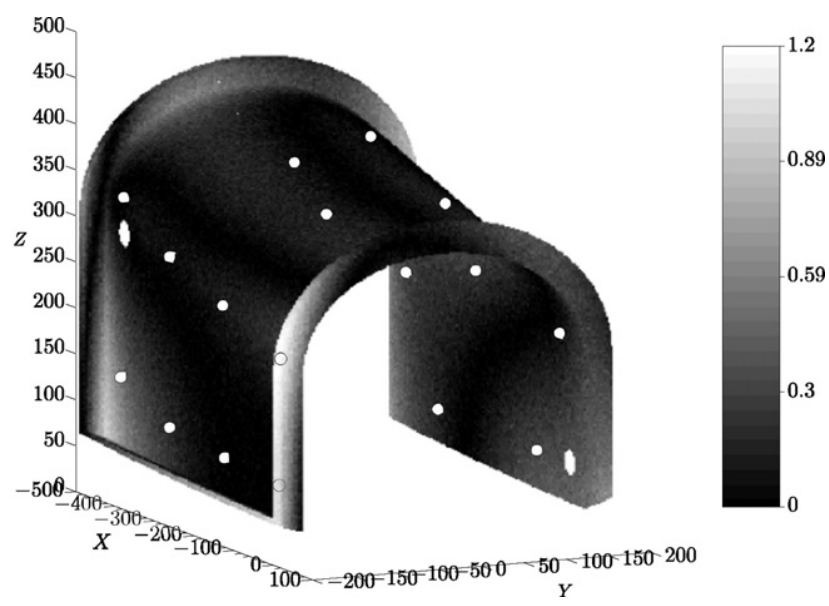

Fig. 12. Actual surface of the landing gear compartment prototype. White dots are the initial design of experiments. The grey levels correspond to the distance values.

\subsection{Iterative strategy results}

For the three structures, a confidence level is defined, the procedure is stopped when $\mathrm{PBC}$ reaches this value. The tolerance value is also depending on the case. Table 2 sums up the characteristic values of each structures.

The confidence level of the landing gear compartment has been raised up because the maximum distance is very small compared

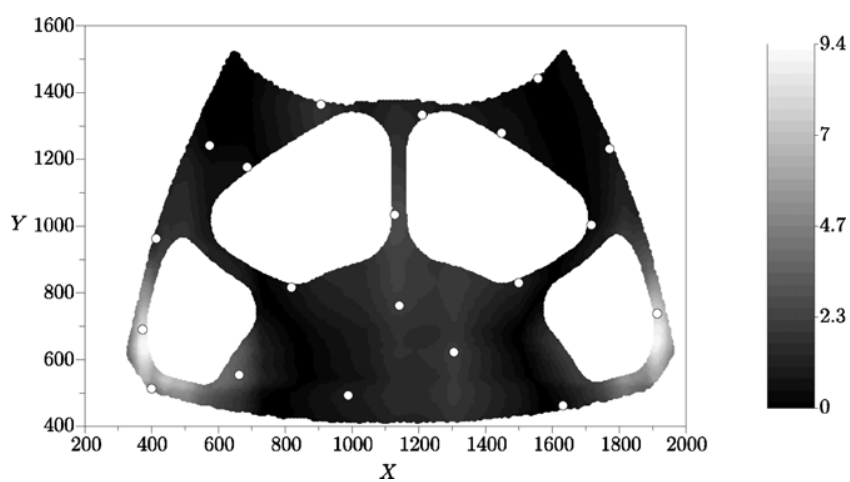

Fig. 13. Initial Kriged cockpit. White dots are the 20 first points of the design of experiments. The grey levels correspond to the distance values. 
Table 2

Characteristic values of each aeronautic structure.

\begin{tabular}{|c|c|c|c|}
\hline & Bulkhead & Cockpit & Landing gear compartment \\
\hline Size of the workpiece $(L \times W \times H \mathrm{~m})$ & $2 \times 1.9 \times 0.2$ & $1.6 \times 1.1 \times 0.66$ & $0.5 \times 0.4 \times 0.42$ \\
\hline Maximum distance from the nominal surface & $25 \mathrm{~mm}$ & $11.25 \mathrm{~mm}$ & $1.18 \mathrm{~mm}$ \\
\hline Considered value of $t / 2$ & $27.5 \mathrm{~mm}$ & $13.1 \mathrm{~mm}$ & $1.3 \mathrm{~mm}$ \\
\hline $\mathrm{CL}$ & 0.997 & 0.997 & 0.99999 \\
\hline
\end{tabular}

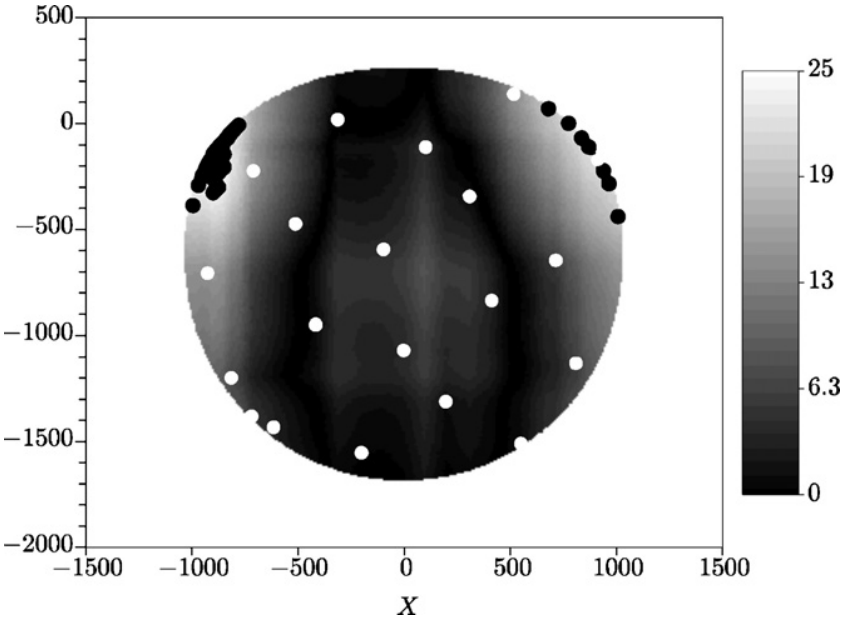

Fig. 14. Final Kriged bulkhead with the entire design of experiments. Black dots are the added points. The grey levels correspond to the distance values.

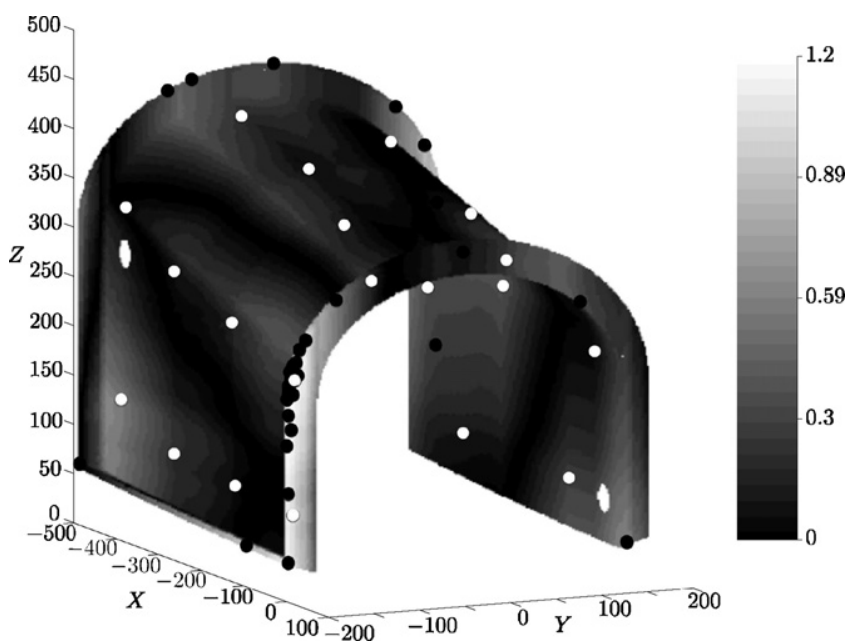

Fig. 15. Final Kriged landing gear compartment with the entire design of experiments. Black dots are the added points. The grey levels correspond to the distance values.

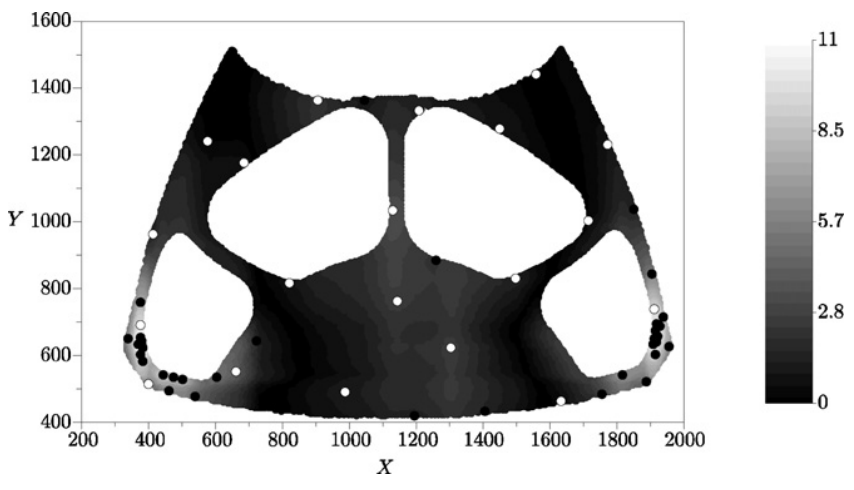

Fig. 16. Final Kriged cockpit with the entire design of experiments. Black dots are the added points. The grey levels correspond to the distance values.
Table 3

Result values of each aeronautic structures.

\begin{tabular}{lccc}
\hline & Bulkhead & Cockpit & $\begin{array}{l}\text { Landing gear } \\
\text { compartment }\end{array}$ \\
\hline Number of iterations & 40 & 36 & 32 \\
Final PBC & 0.9974 & 0.9987 & 0.999996 \\
\hline
\end{tabular}

to the size of the part. In this case, a greater accuracy is required to compensate this gap.

In the three cases, the procedure succeeds in concluding on the conformity of the surfaces with the required probability. Table 3 gives the number of iterations required to reach $\mathrm{CL}$ and the final value of $\mathrm{PBC}$ for each workpiece. The number of required iterations to reach the confidence level is low which proves the efficiency of the proposed procedure.

Figs. 14-16 show the final Kriged surface of the three structures. All the added points are located in the white areas, i.e., where the

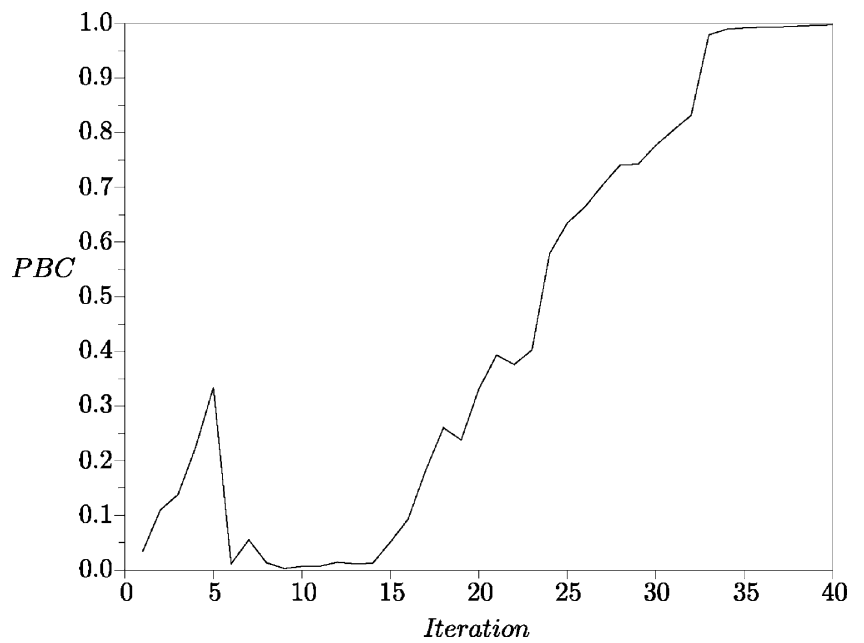

Fig. 17. Convergence of $P B C$ for the bulkhead.

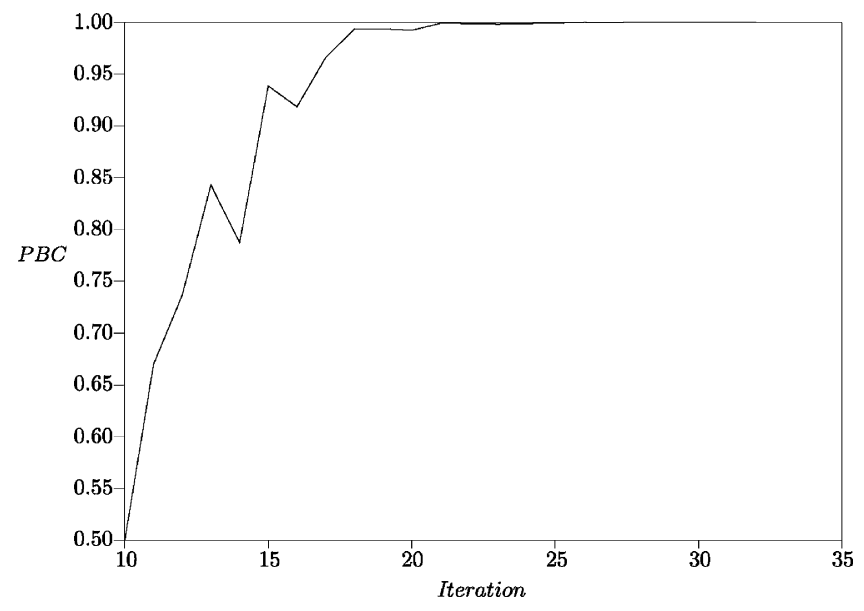

Fig. 18. Convergence of $\mathrm{PBC}$ for the landing gear compartment. 


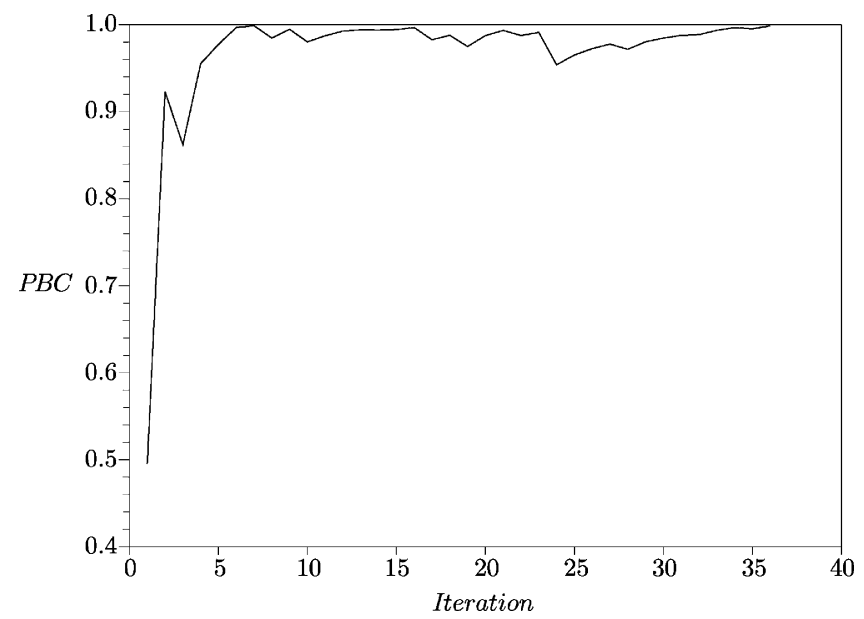

Fig. 19. Convergence of $\mathrm{PBC}$ for the cockpit.

orthogonal distance of the measurements in relation to the nominal geometry is maximum.

The convergence of $\mathrm{PBC}$ of the three structures is shown in Figs. 17-19. In the case of the bulkhead, the finding of the second area at risk leads to the small gap at the fifth iteration in Fig. 17.

\section{Conclusion}

In a competitive economic context where performance requirements are always higher, manufactured products need to be more and more accurate with a limited production cost. Tolerance verification enables to check if products meet the designer's specifications. Measurements are required to verify the conformity of a workpiece. However, a workpiece cannot be perfectly known, which provides several kind of measurement uncertainties. One of these uncertainties is the measurement errors due to the sampling strategy when using a CMM. Particularly, inspection of large workpieces requires lots of measures, the expenses increase proportionately. It is then essential to handle this uncertainty to do more accurate inspections as well as to reduce measurement costs.

This paper presents a procedure called AK-ILS for Active learning method based on Kriging for the Inspection of Large Surfaces. This original approach is based on the Kriging estimation technique. A design of experiments is iteratively improved until the conformity (or not) of the inspected surface is asserted with a confidence level. This method focuses on the measurement errors by measuring a minimum number of relevant points, so the measurement cost is minimised as well. More precisely about the procedure, a Kriging metamodel is built from an initial design of experiments and predictions are made on candidate points to approximate the whole inspected surface. The next measure, selected thanks to a stochastic criterion, is the most probable point outside the tolerance among the candidate points. The iterative process stops either when a required confidence level is reached, the conformity is asserted with such a confidence level, or when a non conform point is found. This procedure is able to deal with profile of a surface whereas most of current solutions cannot. Moreover, the algorithm is easy to implement using the DACE (Design and Analysis of Computer Experiments) Kriging toolbox (http://www2.imm.dtu.dk/ hbn/dace/).

An orientation test case permits to validate the method. Adjusting the tolerance value to be either conform or non conform, the procedure succeeds to conclude, following the case, on the conformity and the non conformity of the tested surface with a little number of measures. After, three nominal geometries of aircraft structures have been disrupted in order to create industrial test cases. The procedure was able to assert the conformity of the three surfaces with less than 60 points, having several thousands of candidate points available to be measured.

Two prospects are considered, the first one is to implement AK-ILS in a CMM software in order to choose in real-time the new measure. The other point is to enhance AK-ILS by taking into account the measurement uncertainty linked to the measuring means of the points.

\section{References}

[1] Kunzmann H, Pfeifer T, Schmitt R, Schwenke H, Weckenmann A. Productive metrology - adding value to manufacture. CIRP Annals - Manufacturing Technology 2005;54(2):155-68.

[2] ISO/TS 17450-2. Geometric Product Specification (GPS) - general concepts part 2: operators and uncertainties. International Organization of Standardization; 2002.

[3] Heping P, Xiangqian J. Evaluation and management procedure of measurement uncertainty in new generation geometrical product specification (GPS). Measurement 2009;42:653-60.

[4] BIPM, IEC, ISO, IUPAC, IUPAP, OIML, Guide to the expression of the uncertainty in measurement, 1st ed. ISBN: 92-6710188-9; 1993.

[5] Weckenmann A, Walz M, Müller W, Eichenberg J. Evaluation of the economical benefit of measurement systems in industrial applications. In: Proceedings of CIRP computer aided tolerancing seminar. 2007.

[6] Colosimo B, Moroni G, Petrò S. A tolerance interval based criterion for optimizing discrete point sampling. Precision Engineering 2010;34:745-54.

[7] McKay M, Beckman R, Conover W. A comparison of three methods for selecting values of input variables in the analysis of output from a computer code. Technometrics 1979;21(2):239-45.

[8] Lee G, Mou J, Shen Y. Sampling strategy design for dimensional measurement of geometric features using coordinate measuring machine. International Journal of Machine Tools and Manufacture 1997;37(7):917-36.

[9] Roth K. Remark concerning integer sequences. Acta Arithmetica 1964;9:257-60.

[10] Lee M-K. A new convex-hull based approach to evaluating flatness tolerance. Computer-Aided Design 1997;29(12):861-8.

[11] Woo T, Liang R, Hsieh C, Lee N. Efficient sampling for surface measurements. Journal of Manufacturing Systems 1995;14(5):345-54.

[12] Prieto F, Redarce T, Lepage R, Boulanger P. An automated inspection system. International Journal of Advanced Manufacturing Technology 2002: 917-25.

[13] Edgeworth R, Wilhelm R. Adaptive sampling for coordinate metrology. Precision Engineering 1999;23:144-54.

[14] Badar M, Raman S, Pulat P. Intelligent search-based selection of sample points for straightness and flatness estimation. Journal of Manufacturing Science and Engineering 2003;125:263-71.

[15] Pedone P, Vicario G, Romano D. Kriging-based sequential inspection plans for coordinate measuring machines. Applied Stochastic Models in Business and Industry 2009;25:133-49.

[16] Mestre M, Abou-Kandil H. Measuring the errors of form of industrial surfaces: prediction and optimization. Precision Engineering 1994;16(4):268-75.

[17] Krige D. A statistical approach to some basic mine valuation problems on the Witwatersrand. Journal of the Chemical, Metallurgical and Mining Society of South Africa 1951;52(6):119-39.

[18] Matheron G. Traité de géostatistique appliquée, vol. 9. Éditions Technip; 1962. 